\title{
BODIPY Dyes for Biomedical Applications: Recent Advances
}

\author{
Seda Cetindere* \\ Department of Chemistry, Gebze Technical University, Turkey \\ *Corresponding author: Seda Cetindere, Department of Chemistry, Gebze Technical University, Gebze, Kocaeli, Turkey. \\ To Cite This Article: Seda Cetindere, BODIPY Dyes for Biomedical Applications: Recent Advances. Am J Biomed Sci \& Res. 2020 - 7(2). AJBSR. \\ MS.ID.001121. DOI: 10.34297/AJBSR.2020.07.001121.
}

Received: 眥 December 10, 2019; Published: 眥 January 29, 2020

\begin{abstract}
In this brief review, some of the recent advances on the development of 4,4-Difluoro-4-bora-3a,4a-diaza-s-indacene (BODIPY) dyes for biomedical applications is presented. Most of the recent studies were focused on fluorescence imaging and Photodynamic Therapy (PDT) and the results of them lead to new approaches in biomedical science.
\end{abstract}

Keywords: BODIPY, Biomedical applications, Fluorescence imaging, PDT

\section{Introduction}

BODIPYs are fluorescent dyes with attention-grabbing properties such as high fluorescence quantum yields (0.6-1.0) and absorption coefficients (60000-80000M-1cm-1), comparatively sharp emission spectra and straightforward functionalization. In addition, they have low sensitivity to each solvent polarity and $\mathrm{pH}$, additionally as wonderful photostability [1-4]. Because of these wonderful properties, BODIPY dyes are utilized in totally different application areas, particularly wide applied in medical specialty areas like biolabeling, bioimaging, PDT etc. [5-7]. This mini review will focus on recent studies about BODIPY dyes in biomedical applications.

In recent years, BODIPY derivatives have been generally applied as fluorescent probes for bioimaging [8-12] and as Photosensitizers (PSs) for PDT [13-17]. Cheng et al. [18] examined the switching mechanism of a Near-Infrared (NIR) aza-BODPIY-based fluorescent dye [18] and its potential usages a Switchable Fluorescent Probe (SFP) in several formats, together with wash-free cell imaging, in vivo tissue imaging, temperature sensing, and tissue UltrasoundSwitchable Fluorescent probe (USF) imaging [19]. These results indicated that this aza-BODIPY based fluorescent dye is highly environment-sensitive dye and showed very weak fluorescence in aqueous solution whereas strong fluorescence in non-polar and high viscosity media. Because of the unique switching property of this dye, it has been successfully applied in wash-free live-cell imaging, fluorescence imaging in live animals, temperature sensing and USF imaging.

Meares et al. [20] synthesized a series of Polyethylene Glycol (PEG) substituted BODIPY-chlorin/bacterochlorin arrays. They examined optical and fluorescence properties of these arrays in organic solvents and aqueous surfactants. They reported two series of arrays: The first series of arrays contains BODIPYs with PEG substituents attached to the boron, whereas in the second series, PEG substituents are attached to the aryl at the meso positions of BODIPY. For both series of arrays, excitation of BODIPY at $500 \mathrm{~nm}$ results in efficient energy transfer to and bright emission of hydro porphyrin in the deep red $(640-660 \mathrm{~nm})$ or near-infrared (740$760 \mathrm{~nm})$ spectral windows. These arrays possess large effective Stokes shift (115-260nm), multiple excitation wavelengths, and narrow, tunable deep red/near-IR fluorescence in aqueous surfactants and reported as promising candidates for a variety of biomedical-related applications [20].

Ogata et al. [21] developed two types of activatable NIR fluorophores derived from bacteriochlorin: chlorin-bacteriochlorin energy transfer dyads and BODIPY-bacteriochlorin energy-transfer dyads. They characterized these fluorophores by multiple narrow excitation bands with comparatively strong emission in NIR. PEG chains were added to enhance the hydrophilicity and decrease aggregation, resulting in a viable in vivo imaging agent that detected 
cancer with high sensitivity in murine models. They reported that these fluorophores supply potential for multicolored fluorescence imaging and are a promising tool for susceptible medicine [21].

Belali et al. [22] have improved a straightforward and efficient way to prepare extremely water-soluble neutral BODIPY formulations by cross-linking them in chitosan-based 3D hydrogel networks. 3,5-diformyl-BODIPY is used as a crosslinker to generate a new class of chitosan-based hydrogels with Fluorescence Resonance Energy Transfer (FRET) dynamics and good solubility in water. The dynamic character of the hydrogel was approved with some rheological, macroscopic and microscopic self-healing tests. The fluorescence lifetime was found to increase in aqueous solution of the BODIPY-chitosan hydrogel compared to the 3,5-diformyl-BODIPY monomer. Experimental results such as red-shift and decreased intensity of the emission spectrum of highly dye-concentrated hydrogel in comparison to dilute hydrogels, together with changes in the fluorescence lifetime of the hydrogel at different concentration of dyes, suggest that the BODIPY-chitosan hydrogels fluorescence dynamics obeyed FRET. They reported that their approach of creating fluorescent hydrogels creates opportunities for new optical polymer designs and uses for biomedical applications such as implantable fluorescent hydrogels [22].

A series of novel fluorescent BODIPY-anionic boron cluster conjugates bearing $\left[\mathrm{B}_{12} \mathrm{H}_{12}\right]^{2}-, \quad\left[3,3^{\prime}-\mathrm{Co}\left(1,2-\mathrm{C}_{2} \mathrm{~B}_{9} \mathrm{H}_{11}\right)_{2}\right]-$, and $\left[3,3^{\prime}-\mathrm{Fe}\left(1,2-\mathrm{C}_{2} \mathrm{~B}_{9} \mathrm{H}_{11}\right)_{2}\right]$-anions have been synthesized from meso(4-hydroxyphenyl) substituted BODIPY by Chaari et al. [23].The syntheses and complete characterization of these compounds were described along with the photophysical properties. Furthermore, the cytotoxicity of the conjugates has been evaluated and their cellular uptake by HeLa cells have been compared by flow cytometry in order to assess their potential as fluorescent dyes for cell tracking. Bioimages of HeLa cells incubated with compounds have been also analyzed by confocal laser microscopy and photoluminescence properties of them have been investigated. Linking anionic boron clusters to the BODIPY does not alter significantly the luminescent properties of the final fluorophores, showing all of them similar emission fluorescent quantum yields (3-6\%). According to the literature, these are the first BODIPY-anionic boron cluster conjugates developed as fluorescent dyes aiming at prospective biomedical applications [23].

Chen et al. [24] investigated the molecular interactions of two 2,6-diiodo-BODIPY derivatives with Human Serum Albumin (HSA) using the combination of experimental and computational studies. Their results demonstrated that the binding to HSA boosted the photodynamic efficiencies of BODIPYs. The BODIPY/HSA complexes exhibited notably enhanced water solubility and singlet oxygen generation efficiency with respect to the BODIPY alone. Additionally, molecular docking, molecular dynamics simulations, and binding free energy calculations provided the structural and energetic insights into the binding mechanism of BODIPY-based derivatives to HSA. According to these results they reported that the formation of complexes of BODIPYs with HSA is a promising strategy for the design and development of BODIPY-based PSs with improved bioavailability and biocompatibility for cancer therapeutic applications [24]

Two PEGylated BODIPY compounds which could self-assemble into Nanoparticles (NPs) in aqueous media were synthesized via Passerini reaction by Zhu et al. [25]. Optical properties including FRET were studied in detail. These results showed that the obtained NPs possess good cytocompatibility and could be used for living cell imaging and effective PDT and shed light on one-pot synthesis of PEGylated fluorescent nanoparticles via multicomponent reaction for biomedical application [25]. Su et al. [26] developed a strategy to fabricate a kind of nanocomposite (CBNPs) via the noncovalent interactions between Carbon Dots (CDs) and BODIPY, by which the solubility and PDT effects of BODIPY were improved through the Fluorescence Resonance Energy Transfer (FRET) mechanism. The CBNPs exhibit good water solubility, excellent singlet oxygen quantum yield, and high biocompatibility and PDT efficiency. They declared that these compounds may have potential applications in biomedical fields and cancer treatment [26].

\section{Conclusion}

According to all these recent studies, it was shown that great advances have been made in biomedical applications especially on living cell imaging and PDT, with using BODIPY dyes due to their excellent photophysical and photochemical properties. Soon, its waited for the studies with these dyes in biomedical applications are expected to potentially increase.

\section{References}

1. Ulrich G, Ziessel R, Harriman A (2008) The chemistry of fluorescent bodipy dyes: versatility unsurpassed. Angew Chem Int Ed 47(7): 11841201.

2. Zhang C, Zhao J, Wu S, Wang Z, Wu W, et al. (2013) Intramolecular RET enhanced visible light-absorbing bodipy organic triplet photosensitizers and application in photooxidation and triplet-triplet annihilation up conversion. J Am Chem Soc 135(28): 10566-10578.

3. Kowada T, Maeda H, Kikuchi K (2015) BODIPY-based probes for the fluorescence imaging of biomolecules in living cells. Chem Soc Rev 44: 4953-4972.

4. Turksoy A, Yildiz D, Akkaya EU (2019) Photosensitization and controlled photosensitization with BODIPY dyes. Coord Chem Rev 379: 47-64.

5. Ni Y, Wu J (2014) Far-red and near infrared BODIPY dyes: synthesis and applications for fluorescent $\mathrm{pH}$ probes and bio-imaging. Org Biomol Chem 12: 3774-3791.

6. Kue CS, Ng SY, Voon SH, Kamkaew A, Chung LY, et al. (2018) Recent strategies to improve boron dipyrromethene (BODIPY) for photodynamic cancer therapy: an updated review. Photochem Photobiol Sci 17(11): 1691-1708.

7. Zhang T, Ma C, Sun T, Xie Z (2013) Unadulterated BODIPY nanoparticles for biomedical applications. Coordination Chemistry Reviews 390: 7685. 
8. Georgiev NI, Bryaskova R, Tzoneva R, Ugrinova I, Detrembleur C, et al (2013) A novel pH sensitive water soluble fluorescent nanomicellar sensor for potential biomedical applications. Bioorganic \& Medicinal Chemistry 21(21): 6292-6302.

9. Zatsikha YV, Yakubovskyi VP, Shandura MP, Dubey IY, Kovtun YP (2013) An efficient method of chemical modification of bodıpy core. Tetrahedron 69: 2233-2238.

10. Zatsikha YV, Yakubovskyi VP, Shandura MP, Dubey IY, Kovtun YP (2015) Functionalized bispyridoneannelated BODIPY - Bright long-wavelength fluorophores. Dyes and Pigments 114: 215-221.

11. Shen BX, Qian Y (2017) Triphenylamine-bodıpy fluorescent dendron: click synthesis and fluorometric chemodosimeter for $\mathrm{Hg} 2+, \mathrm{Fe} 3+$ based on the $\mathrm{C}=\mathrm{N}$ Bond. ChemistrySelect 2(8): 2406-2413.

12. Shimizu K, Kitagawa D, Kobatake S (2013) Solid emission color tuning of polymers consisting of bodipy and styrene in various ratios. Dyes and Pigments 161: 341-346.

13. Verwilst P, David CC, Leena V, Hofkens J, de Witte PAM, et al. (2013) Synthesis and in vitro evaluation of a PDT active BODIPY-NLS conjugate. Bioorganic \& Medicinal Chemistry Letters 23(11): 3204-3207.

14. Kamkaew A, Burgess K (2013) Double-Targeting Using a TrkC Ligand Conjugated to Dipyrromethene boron Difluoride (BODIPY) Based Photodynamic Therapy (PDT) Agent. Med Chem 56(19): 7608-7614.

15. Lu Z, Zhang X, Zhao Y, Xue Y, Zhai T, et al. (2015) BoDIPY-based macromolecular photosensitizer with cation-enhanced antibacterial activity. Polym Chem 6: 302-310.

16. Mazzone G, Domenico A, Russo QN (2016) PDT-correlated photophysical properties of thienopyrrole BODIPY derivatives. Theoretical insights. Dyes and Pigments 130: 9-15.

17. Zagami R, Sortino G, Caruso E, Malacarne MC, Banfi S, et al. (2018) Tailored-BODIPY/amphiphilic cyclodextrin nano assemblies with PDT effectiveness. Langmuir 34(29): 8639-8651.

18. Cheng B, Bandi V, Wei MY, Pei Y, D’Souza F, et al. (2016) High-resolution ultrasound-switchable fluorescence imaging in centimeter-deep tissue phantoms with high signal-to-noise ratio and high sensitivity via nove contrast agents. PLoS ONE 11(11): 0165963.

19. Cheng B, Bandi V, Yu S, D’Souza F, Nguyen KT, et al. (2017) The mechanisms and biomedical applications of an NIR bodıpy-based switchable fluorescent probe. Int J Mol Sci 18(2): 384-398.

20. Meares A, Satraitis A, Akhigbe J, Santhanam N, Swaminathan S, et al (2017) Amphiphilic bodıpy-hydroporphyrin energy transfer arrays with broadly tunable absorption and deep red/near-infrared emission in aqueous micelles. J Org Chem 82(12): 6054-6070.

21. Ogata F, Nagaya T, Maruoka Y, Akhigbe J, Meares A, et al. (2019) Activatable near-infrared fluorescence imaging using PEGylated bacteriochlorin-based chlorin and bodipy-dyads as probes for detecting cancer. Bioconjugate Chem 30(1): 169-183

22. Belali S, Emandi G, Cafolla AA, O'Connell B, Haffner B, et al. (2017) Water-soluble, neutral 3,5-diformyl-BODIPY with extended fluorescence lifetime in a self-healable chitosan hydrogel. Photochem Photobiol Sci 16: $1700-1708$

23. Chaari M, Gaztelumendi N, González JC, Moledo PP, Viñas C, et al. (2018) Fluorescent BODIPY-Anionic Boron Cluster Conjugates as Potential Agents for Cell Tracking. Bioconjugate Chem 29(5): 1763-1773.

24. Chen Y, Liu J, Song M, Jiang L, Liu L, et al. (2018) Insights into the binding mechanism of BODIPY-based photosensitizers to human serum albumin: A combined experimental and computational study. Spectrochimica Acta Part A: Molecular and Biomolecular Spectroscopy 203: 158-165.

25. Zhu Y, Lin W, Zhang W, Feng Y, Wu Z, et al. (2017) PEGylated BODIPY assembling fluorescent nanoparticles for photodynamic therapy. Chinese Chemical Letters 28(9): 1875-1877.

26.Su Y, Lu S, Gao P, Zheng M, Xie Z (2019) BODIPY@carbon dot nanocomposites for enhanced photodynamic activity. Mater Chem Front 3: $1747-1753$ 